\title{
Two-Photon Absorption Spectroscopy using Intense Phase-Chirped Entangled Beams
}

\author{
Jiří Svozilík \\ Quantum Optics Laboratory, Universidad de los Andes, A.A. 4976, Bogotá D.C., Colombia \\ Joint Laboratory of Optics of Palacký University and Institute of Physics of CAS, \\ 17. listopadu 12, 77146 Olomouc, Czech Republic \\ Jan Peřina Jr. \\ Joint Laboratory of Optics of Palacký University and Institute of Physics of CAS, \\ 17. listopadu 12, 77146 Olomouc, Czech Republic \\ Roberto de J. León-Montiel \\ Instituto de Ciencias Nucleares, Universidad Nacional Autónoma de México, Apartado \\ Postal 70-543, 04510 Cd. Mx., México
}

\begin{abstract}
We numerically analyze the use of intense entangled twin beams for ultrasensitive spectroscopic measurements in chemical and biological systems. The examined scheme makes use of intense frequency-modulated (chirped) entangled beams to successfully extract information about the intermediate material states that contribute to the two-photon excitation of an absorbing medium. Robustness of the presented method is examined with respect to the applied intervals of the frequency chirp.
\end{abstract}

\section{Introduction}

Quantum entanglement, which lies at the heart of modern quantum spectroscopy [1, 2, 3] has been recognized as a powerful resource for the development of novel methods and applications in various fields of research, including quantum cryptography [4], quantum computing [5], and quantum metrology [6]. In particular, regarding the latter, the use of entangled light in two-photon absorption (TPA) spectroscopy has already received a great deal of attention 7, $8,[9,10,11,12,13$ because of the unique phenomena that arise in the interaction of entangled photon pairs with matter. As examples, we mention linear scaling of the TPA rates on the photon flux [14], two-photon-induced transparency [15], the ability to select different states in complex biological aggregates [16], and the control of entanglement in matter [17, 18]. Indeed, the prediction and observation of these fascinating effects can be understood as a 
direct consequence of the dependence of the TPA signal on the properties of quantum light that interacts with the sample [19, 20, 21].

Among different techniques proposed over the years, entangled-photon based spectroscopy techniques such as the virtual-state (VSS) and pump-probe (PPS) spectroscopies [1, 22, 2, 23, 3] have proved to be a unique tool for extracting information about the intermediate levels [24, 25], that contribute to the twophoton excitation of an absorbing medium. In these techniques, intermediate transitions, a signature of the medium, are experimentally revealed by introducing a time delay between photons comprising a state entangled in frequencies.

Regardless of some experimental issues expressed in the original proposal of VSS, it can be considered as a new route towards novel applications in ultrasensitive detection [26]. In this contribution, we propose an experimental scheme that can successfully overcome two issues. Generally speaking, quantum based spectroscopic methods are usually experimentally challenging due to the low TPA rates and required weak sources of photon pairs. This first issue is addressed by considering that the medium interacts with a twin beam composed by a larger number of entangled photon pairs and generated by means of intense parametric down-conversion (PDC) [27, 28, 29]. Indeed, properties of such twin beams have been commonly investigated [30, 31, 32] and, more importantly, it has been shown that strong quantum frequency correlations inside the twin beam persist even at these higher photon-flux conditions as shown in 33. and also later in [34, 35, 36]. A 3D model of an intense twin beam developed in 37] has revealed that typically tens of independent dual spectral Schmidt modes are found in such intense twin beams in quantum superpositions. For ultrashort pump pulses, these Schmidt modes are delocalized in the whole twinbeam spectral range which guarantees the performance of the VSS in the whole used spectral region. Though the weights of individual members in these quantum superposition change with the increasing twin-beam intensity, there still remains sufficient numbers of comparably populated members that keep quantum character of the twin-beam state that allow for the VSS. The use of such twin beams in the quantum spectroscopy has been recently discussed in [38].

The second issue comes with the necessity of averaging over several realizations differing in temporal correlations (entanglement) between the photons obtained by either changing the width of the pump pulse (in Type-I nonlinear process) or the length of the nonlinear crystal (Type-II) in each realization [1], which can be experimentally cumbersome. This approach is required to correctly identify the transition frequencies in a detected signal from their combinations. We note that the detected signal encompasses contributions from all possible quantum paths that lead to the absorption of two photons with the participation of two arbitrary intermediate levels. However, only the paths at which the same intermediate level is used give us the looked-for energies of the intermediate levels. Alternatively to the discussed averaging approaches, we may vary and control the properties of entangled photon pairs by modifying the spatial shape of the pump beam [39, 40]. To address this issue, we make use of a technique in which the spectral entanglement between photons is modified by frequency variations in the spectrum of the signal beam. Such approach 
has been extensively studied in Refs. [41, 42, 43]. Because typical correlation times of down-converted photons are found in the femtosecond domain, one needs to make sure that any frequency modulation of photons is done in the ultrafast regime. Such methods already exist, in particular those based on the use of spatial light modulators, which have shown to be extremely efficient for femtosecond pulse shaping [43].

Here we show that this technique can also be used to extract information about the energy-level structure of the sample by computing the relative variance of several TPA signals obtained for different signal frequency chirps, as depicted in Fig. 1. Robustness of the proposed method, which is an important practical issue, is also discussed later.

Our proposed scheme might open a new avenue towards the experimental implementation of quantum spectroscopy by overcoming the low photon-flux issue, while maintaining the most important feature of this technique which lies in the fact that, unlike other commonly used TPA spectroscopy techniques that require sophisticated tunable sources, it can be implemented by carrying out pulsed or continuous-wave absorption measurements without changing the wavelength of the source [1, 44, 2]. The use of spectrally broad-band photon-pair fields, that may cover even 2-3 hundreds of $\mathrm{nm}$ in the visible spectral range, together with the fact that VSS scans the whole spectral range in each measurement repetition make the VSS in principle superior above the other conventionally used spectroscopic methods with tunable light sources. We note that the effect of scanning the whole spectral range in each experimental repetition is reached also when two ultrashort pulses with exactly opposed chirps are applied to observe twophoton absorption, as suggested in [16]. However, practical realization of this method would require perfect synchronization of both chirps and moreover the covered spectral range, that is derived from the pulses spectral widths, would be quite narrow.

\section{Theoretical background}

Let us consider the interaction of a medium with a twin beam, described by the interaction Hamiltonian $\hat{H}_{I}(t)=\hat{\mu}(t) \hat{E}^{(+)}(t)$, where $\hat{\mu}(t)$ is the medium dipole-moment operator and $\hat{E}^{(+)}(t)=\hat{E}_{s}^{(+)}(t+\tau)+\hat{E}_{i}^{(+)}(t)$ is the overall positive-frequency electric-field operator, with subscripts $s$ and $i$ denoting the signal and idler fields that constitute the twin beam. Notice that we have included a time delay $\tau$ between the fields, which is needed for implementing the pump-probe technique.

We assume that the medium is initially in its ground state $|g\rangle$ (with energy $\left.\epsilon_{g}\right)$. Then, the probability that the medium is excited into its final state $|f\rangle$ (with energy $\epsilon_{f}$ ), through a TPA process, is obtained by means of second-order time-dependent perturbation theory in the form [22, 45, 46]:

$$
\begin{aligned}
P_{g \rightarrow f} & =\int_{-\infty}^{\infty} d t_{2} \int_{-\infty}^{t_{2}} d t_{1} \int_{-\infty}^{\infty} d t_{2}^{\prime} \int_{-\infty}^{t_{2}^{\prime}} d t_{1}^{\prime} M^{*}\left(t_{2}, t_{1}\right) \\
& \times G^{(2)}\left(t_{2}, t_{1} ; t_{2}^{\prime}, t_{1}^{\prime}\right) M\left(t_{2}^{\prime}, t_{1}^{\prime}\right),
\end{aligned}
$$




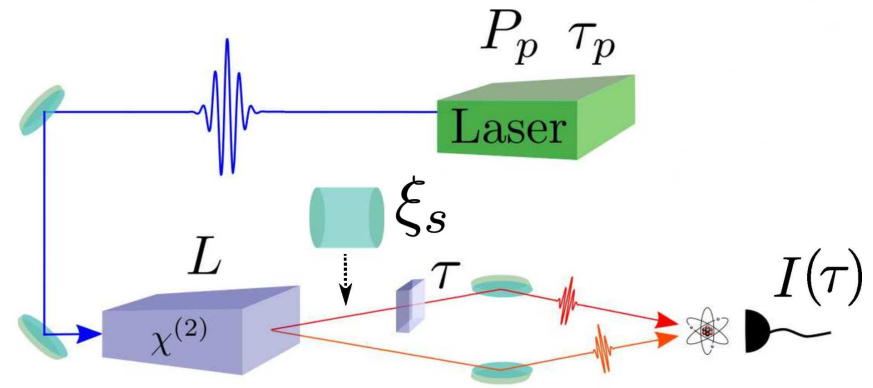

Figure 1: Proposed experimental setup. A pump pulse, with time duration $\tau_{p}$ and power $P_{p}$, interacts with a nonlinear crystal of length $L$, which serves as a source of twin beams composed of many photon pairs. The generated photons are spatially separated and a time delay $\tau$ between them is introduced. Information about virtual transitions is then obtained by monitoring the two-photon absorption rate as a function of delay $\tau$. In order to obtain the relative variances of resolved spectral peaks, the signal-beam spectrum is modulated by a phase modulator generating a frequency chirp $\xi_{s}$.

where $M$ stands for the two-point temporal dipole-moment correlation function,

$$
M\left(t_{2}, t_{1}\right)=\sum_{j} \frac{\mu_{f j} \mu_{j g}}{\hbar^{2}} \exp \left[i\left(\epsilon_{f}-\epsilon_{j}\right) t_{2}+i\left(\epsilon_{j}-\epsilon_{g}\right) t_{1}\right],
$$

$G^{(2)}$ denotes the optical-field fourth-order

$$
G^{(2)}\left(t_{2}, t_{1} ; t_{2}^{\prime}, t_{1}^{\prime}\right)=\left\langle\hat{E}^{(-)}\left(t_{2}\right) \hat{E}^{(-)}\left(t_{1}\right) \hat{E}^{(+)}\left(t_{2}^{\prime}\right) \hat{E}^{(+)}\left(t_{1}^{\prime}\right)\right\rangle,
$$

and $\hbar$ stands for the reduced Planck constant.

Equation (11) describes the response of the medium to the applied twin beam and $\mu_{f j}$ and $\mu_{j g}$ are the transition matrix elements of the dipole-moment operator between the states specified in the subscript. Notice that the excitation of the medium occurs through intermediate, fast decaying, states $|j\rangle$, with complex energy eigenvalues $\epsilon_{j}=\tilde{\epsilon}_{j}-i \kappa_{j}$; $\tilde{\epsilon}_{j}$ is the energy eigenvalue of the $j$ th intermediate level and $\kappa_{j}$ gives its natural line-width.

On the other hand, Eq. (2), giving the fourth-order field correlation function [47, 48], characterizes statistical properties of the interacting optical fields. In what follows, we concentrate on the description of the quantum fields comprising the twin beam, whose temporal (as well as spectral) correlations are controlled by frequency variations in the spectrum of the signal field. We note that these correlations are by definition quantum as they describe the properties of spectral quantum superpositions of the Schmidt dual modes comprising the twin beam (see below). The intense twin beams composed of many photon pairs can be conveniently described by using the two-step procedure [35, 37, 49, 33, 38]. 
Photon fields occurring in Eq. (20) are described by the field operators [50]:

$$
\begin{aligned}
\hat{E}_{j}^{(+)}(z, t) & =\int d \omega_{j} \sqrt{\frac{\hbar \omega_{j}}{2 \pi c A_{q} n_{j}}} \hat{a}_{j}\left(z, \omega_{j}\right) \\
& \times \exp \left[-i\left(\omega_{j} t-k_{z j}\left(\omega_{j}\right) z\right)\right], \quad j=s, i ;
\end{aligned}
$$

$n_{j}$ is the index of refraction of field $j$, and $\hat{a}_{j}\left(z, \omega_{j}\right)$ stands for the photon annihilation operator of a mode with frequency $\omega_{j}$. The corresponding longitudinal wave-vector is denoted as $k_{z j}$. $A_{q}$ is the quantization area.

The standard procedure for obtaining the quantum state of photon twin beams, that is based on the first-order perturbation solution of the Schrödinger equation, results in the following spectrally entangled two-photon state $|\Phi\rangle$ :

$$
|\Phi\rangle=\int_{-\infty}^{\infty} d \omega_{s} \int_{-\infty}^{\infty} d \omega_{i} \Phi\left(\omega_{s}, \omega_{i}\right) \hat{a}_{s}^{\dagger}\left(\omega_{s}\right) \hat{a}_{i}^{\dagger}\left(\omega_{i}\right)|\mathrm{vac}\rangle
$$

The two-photon spectral amplitude $\Phi\left(\omega_{s}, \omega_{i}\right)$ introduced in Eq. (4) is equal to:

$$
\begin{aligned}
& \Phi\left(\omega_{s}, \omega_{i}\right)=C_{\Phi} \sqrt{\omega_{s} \omega_{i}} \exp \left[-\frac{\tau_{p}^{2}}{4}\left(\omega_{s}+\omega_{i}-\omega_{p}^{0}\right)^{2}\right] \\
& \times \int_{-L}^{0} d z \exp \left(i\left[k_{z p}\left(\omega_{s}+\omega_{i}\right)-k_{z s}\left(\omega_{s}\right)-k_{z i}\left(\omega_{i}\right)\right] z\right) .
\end{aligned}
$$

where the constant $C_{\Phi}=\frac{2 i \chi^{(2)} \eta_{p}}{\sqrt{2 \pi n_{p} n_{s} n_{i}}} \sqrt{\frac{\tau_{p}}{\sqrt{2 \pi}}}$. The pump-pulse duration is denoted as $\tau_{p}$. The parameter $\eta_{p}=\sqrt{P_{p} / \epsilon_{0} c f n_{p}}$ quantifies the pump amplitude, which depends on pump power $P_{p}$, pump repetition rate $f$, medium index of refraction $n_{p}$, and the speed of light $c$. The central frequency of the pump field is denoted as $\omega_{p}^{0}$. The nonlinear medium of length $\mathrm{L}$ is characterized via the nonlinear susceptibility $\xi^{(2)}$. The Schmidt decomposition of the normalized two-photon spectral amplitude $\tilde{\Phi}, \tilde{\Phi}=\Phi / \mathcal{N}$ and $\mathcal{N}^{2}=\int d \omega_{s} \int d \omega_{i}\left|\Phi\left(\omega_{s}, \omega_{i}\right)\right|^{2}$, reveals pairs of spectral modes [51, 52] obeying the relation:

$$
\tilde{\Phi}\left(\omega_{s}, \omega_{i}\right)=\sum_{g=1}^{\infty} \lambda_{g} f_{s, g}^{*}\left(\omega_{s}\right) f_{i, g}^{*}\left(\omega_{i}\right)
$$

Here $\left\{\lambda_{g}\right\}_{g=1}^{\infty}$ is the set of singular values belonging to singular vectors $\left\{f_{s, g}\left(\omega_{s}\right)\right\}_{g=1}^{\infty}$ and $\left\{f_{i, g}\left(\omega_{i}\right)\right\}_{g=1}^{\infty}$. Then the creation operators of Schmidt modes $\hat{a}_{s, g}^{\dagger}\left[\hat{a}_{i, g}^{\dagger}\right]$ for the signal (idler) eigenfunction $f_{s, g}\left(\omega_{s}\right)\left[f_{i, g}\left(\omega_{i}\right)\right]$ are defined by the formula:

$$
\hat{a}_{j, g}^{\dagger}=\int_{-\infty}^{\infty} f_{j, g}^{*}\left(\omega_{j}\right) \hat{a}_{j}^{\dagger}\left(\omega_{j}\right), \quad j=s, i
$$

The inverse relation attains the form:

$$
\hat{a}_{j}^{\dagger}\left(\omega_{j}\right)=\sum_{g=1}^{\infty} f_{j, g}\left(\omega_{j}\right) \hat{a}_{j, g}^{\dagger} .
$$


These Schmidt modes enter to the Heisenberg equations [33, 35] to obtain the solutions for monochromatic fields:

$$
\hat{a}_{s}\left(\omega_{s}, L\right)=\sum_{g=1}^{\infty} f_{s, g}^{*}\left(\omega_{s}\right)\left[u_{g} \hat{a}_{s, g}(0)+v_{g} \hat{a}_{i, g}^{\dagger}(0)\right] .
$$

here $u_{g}=\cosh \left(\mathcal{N} \lambda_{g}\right)$ and $v_{g}=\sinh \left(\mathcal{N} \lambda_{g}\right)$. Substitution of Eq. (8) into Eq (3) giving the signal and idler electric-field amplitudes $\hat{E}_{j}^{(+)}(z=L, t), j=$ $s, i$, allows us to evaluate the optical-field fourth-order correlation function $G^{(2)}\left(t_{1}, t_{2}, t_{1}^{\prime}, t_{2}^{\prime}\right)$ defined in Eq. (2).

To identify the correct intermediate levels among all spectral peaks, we introduce a quadratic frequency chirp, controlled by the parameter $\xi_{s}$, to the signal-photon path (or, equivalently, to the idler-photon path). This is accomplished by transforming the signal-photon operators given by Eq. (8) in the following way

$$
\hat{a}_{s}\left(z, \omega_{s}\right) \rightarrow \exp \left[i \xi_{s}\left(\omega_{s}-\omega_{s 0}\right)^{2}\right] \hat{a}_{s}\left(z, \omega_{s}\right) .
$$

Finally, the mean photon-pair number $N_{j}$ of the twin beam is determined by $N_{j}=\int d \omega_{j}\left\langle\hat{a}_{j}^{\dagger}\left(\omega_{j}\right) \hat{a}_{j}\left(\omega_{j}\right)\right\rangle=\sum_{g=1}^{\infty}\left\langle\hat{a}_{j, g}^{\dagger} \hat{a}_{j, g}\right\rangle=\sum_{g=1}^{\infty}\left|v_{g}\right|^{2}$. This means that in order to have intense twin beams, the condition $N_{j}=\sum_{g=1}^{\infty}\left|v_{g}\right|^{2} \gg 1$ has to be satisfied.

\section{Numerical Results}

With the aim of demonstrating the capability of the proposed scheme for extracting information about the energy level structure of the medium, we consider a model system whose two-photon transitions take place via three intermediate states with randomly-chosen energies $\epsilon_{j}=\{1.586,1.604,1.619\} \mathrm{eV}$ and the doubly-excited state satisfying the condition $\epsilon_{f}-\epsilon_{g}=3.0996 \mathrm{eV}$. For the simplicity, we set all transition dipole moments $\mu_{f k}$ and $\mu_{k g}$ such $\mu_{f k} \mu_{k g} / \hbar^{2}=1$ $\mathrm{m}^{2} \cdot \mathrm{V}^{-2} \cdot \mathrm{s}^{-2}$. In addition, we assume the intermediate-state lifetimes much larger than the twin-beam time duration as well as the relative delay $\tau$ [1, 44, 2]. As the twin-beam source, we consider a near-to-collinear PDC with Type-II interaction, where a nonlinear crystal, of length $L=0.801 \mathrm{~mm}$ and with inverse group velocities $G_{p}=5.4 \mathrm{ps} / \mathrm{m}, G_{s}=5.2 \mathrm{ps} / \mathrm{m}, G_{i}=5.6 \mathrm{ps} / \mathrm{m}$ of the pump, signal, and idler photons, is illuminated by a laser pulse of wavelength $\lambda_{p}=0.4 \mu \mathrm{m}$ with time duration $\tau_{p}=1 \mathrm{ps}$. For the sake of simplicity, we assume a spectrally degenerate twin beam, i.e. $\lambda_{s}=\lambda_{i}=\lambda_{p} / 2$.

Figure 2(a) shows the TPA transition signal [Eq. (11)] as a function of the delay between the pulses, each of which carrying $N_{j} \approx 100$ photons. The TPA transition signal is normalized with respect to its maximal value in the whole range of $\tau$. Notice the non-monotonic behaviour of the TPA transition probability. This phenomenon, related to the entanglement-induced two-photon transparency [15], arises from the quantum interference between different interaction pathways, shown in Figure 3, through which two-photon excitation of 


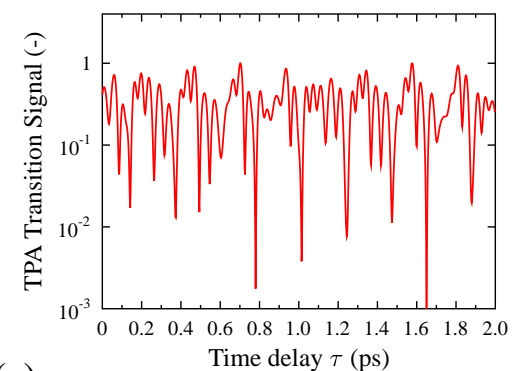

(a)

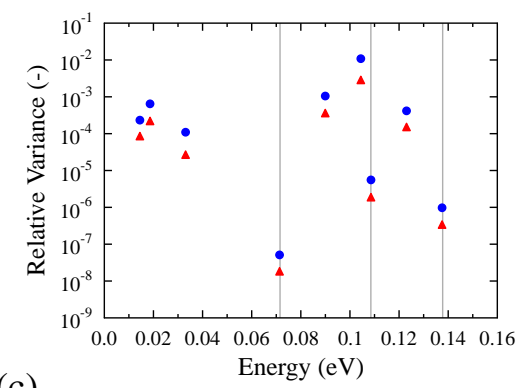

(c)

Figure 2: (a) TPA transition signal as a function of the delay $\tau$ between fields carrying $N_{j} \approx 100$ photons. Spectral decomposition [Eq. [5] of the twin beams was done using 500 singular vectors. (b) Fourier transform of the TPA transition probability shown in (a). (c) Relative variance of transition-probability fluctuations of the peaks resolved in the TPA spectra obtained for 20 different values of the signal-frequency chirp $\xi_{s} \in\langle 0.0,9.5\rangle \mathrm{fs}^{2}$ (blue circles) and 40 values $\xi_{s} \in\langle 0.0,3.9\rangle \mathrm{fs}^{2}$ (red triangles). The crystal has the length $L=$ $0.801 \mathrm{~mm}$. (d) Spectrum of the TPA transition probability averaged over an ensemble of 100 crystals of different lengths $L \in\langle 20,22\rangle \mathrm{mm}$. The vertical grey lines indicate the doubled relative energies $\left|2 \epsilon_{j}-\epsilon_{f}\right|$ of the intermediate levels.

the medium can occur. To retrieve the spectroscopic information present in the TPA signal, one might feel tempted to directly Fourier transform Eq. (1). However, as pointed out in Ref. 1], the TPA signal as a function of delay $\tau$ contains spectral components at various intermediate frequencies that hinder the clear identification of intermediate transitions [see Figures [2(a,b) and 4 ]. To address this issue, we introduce a new method that benefits from different strengths of transition probability fluctuations characterizing the sought and undesired peaks, which originate from the twin-beam spectral changes induced by the signal-frequency chirp $\xi_{s}$. These fluctuations are quantified by the relative variance $R_{n}$ defined as:

$$
R_{n}=\frac{\operatorname{var}_{\xi_{\mathrm{s}}}\left[\mathrm{P}_{\mathrm{n}}\right]}{M_{\xi_{s}}\left[P_{n}\right]^{2}}
$$

where $P_{n}$ is the transition probability of the $n$th spectral peak, and $M_{\xi_{s}}\left[P_{n}\right]$ 
(b)

(a)

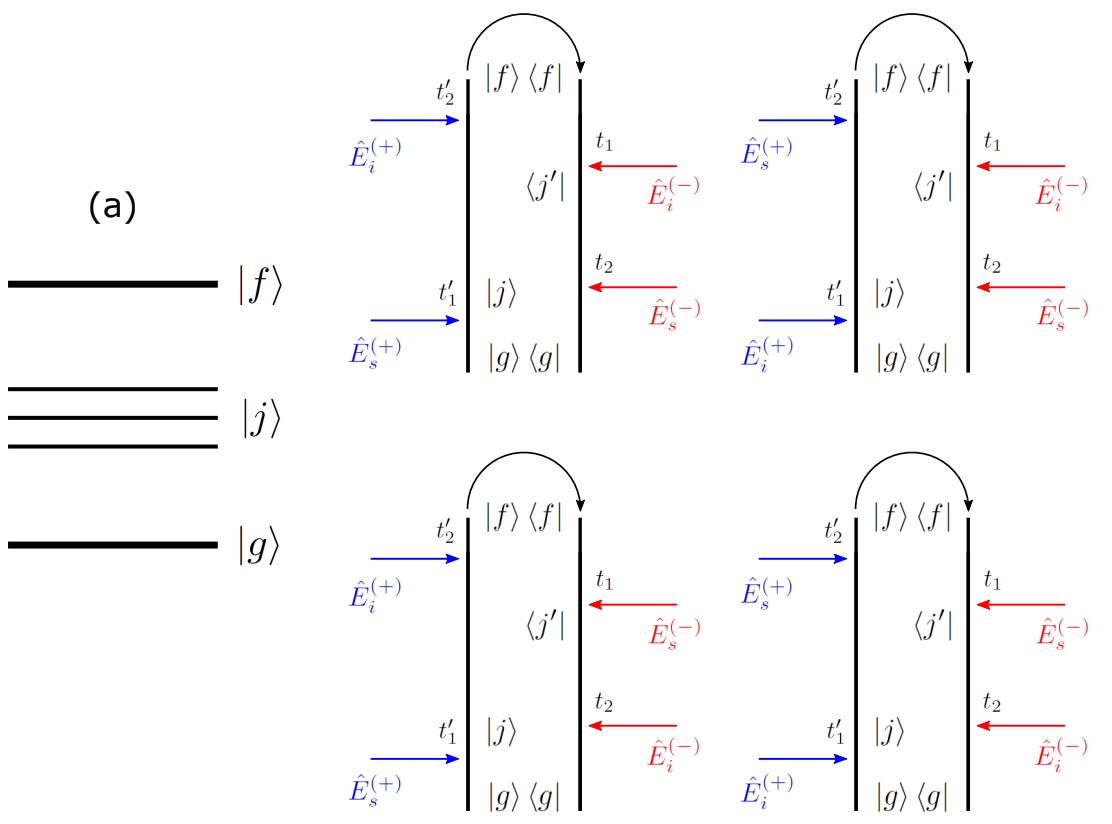

Figure 3: (a) Scheme of energy levels used in our simulations. (b) Closed time path loop diagrams 9, 53], representing the entangled photon-matter quantum interaction pathways that contribute to the non-monotonic behaviour of the TPA signal. The time-ordered photonabsorption events can be seen by moving clockwise along the loops.

its mean value. Figure 2(c) shows the relative variance of the resolved peaks present in the spectrum of the TPA signal [Fig. 2(b)]. It is clearly visible that the peaks with the lowest fluctuations (marked with vertical gray lines, notice the vertical logarithmic scale) are located at the sought energies $\left|2 \epsilon_{j}-\epsilon_{f}\right|$. This allows us to immediately identify the intermediate states contributing to the two-photon excitation of the medium. The success of this technique resides in the fact that the signal-frequency chirp does not disturb probabilities of these peaks, whereas the probabilities of the rest of the peaks, that are undesired, strongly fluctuate around their mean values.

To compare our approach with the original proposal [1], we perform in parallel an average of the TPA signal over different crystal lengths. The obtained spectrum of the averaged signal is plotted in Fig. 2(d). In the spectrum, the positions of the peaks that emerge from the Fourier transform of the averaged TPA signal exactly match those identified in our approach by the lowest available fluctuations. This demonstrates that our spectroscopic method can successfully retrieve information about the intermediate-state energies. Importantly, in contrast to the original implementation of VSS, the average is done by simply changing the chirp introduced into the signal beam. 


\section{Robustness of the proposed scheme}

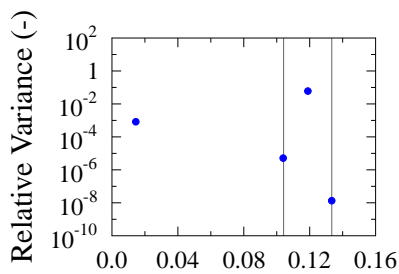

(a)

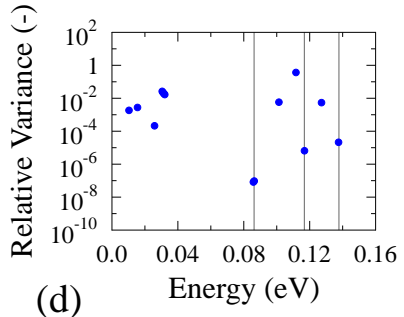

(d)

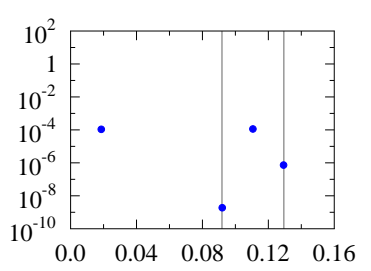

(b)

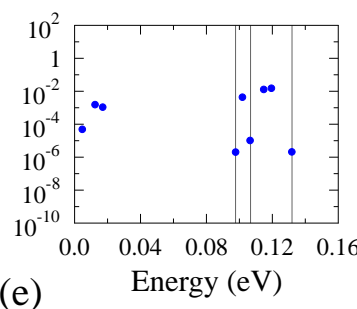

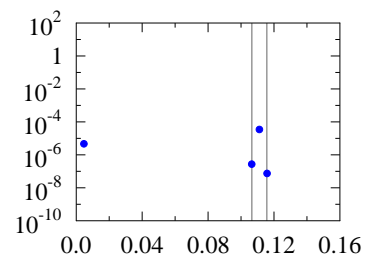

(c)

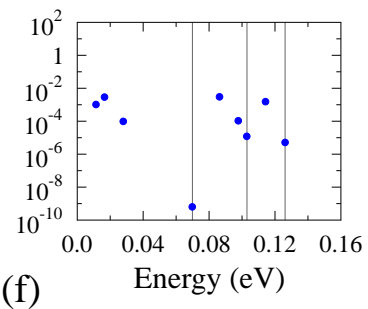

Figure 4: Relative variances originating from (a-c) 2-intermediate levels and (d-f) 3 intermediate levels considering 20 steps of the chirp parameter $\xi_{s}$ in the interval $\langle 0,19\rangle \mathrm{fs}^{2}$. The used crystal length equals (a) $0.8 \mu \mathrm{m}$, (b) $0.55 \mu \mathrm{m}$, (c) $0.65 \mu \mathrm{m}$, (d) $0.8 \mu \mathrm{m}$, (e) $0.8 \mu \mathrm{m}$, and (f) $0.716 \mu \mathrm{m}$.

Since a full-scale proof of the presented method is numerically highly demanding, we show the application of our approach on additional examples of model systems having 2 and 3 intermediate levels with random energies. For each configuration, a suitable crystal length has been used and subsequently calculations of TPA signals for varying $\xi_{s}$ have been performed. From the ob-

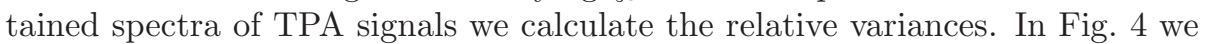
show the results for different intermediate-level configurations. Plots (a-c) of the first row of Fig. 4 correspond to the case with 2 intermediate levels, whereas the plots (d-f) in the second row show the cases with 3 intermediate levels. In all presented cases, the amount of signal photons have been maintained in the regime where the contributions originating in the quantum field entanglement prevail.

Notice that the results presented in Fig. 4 show that the energy levels of the sample appear as those with the minimal relative variances, thus providing a simple way to characterize the electronic-level structure of an unknown sample.

\section{Conclusion}

A new scheme for the implementation of quantum entangled spectroscopy has been suggested and analyzed. The proposed scheme makes use of intense twin beams with the spectral chirp introduced to the signal beam. With 
our technique, we were able to successfully retrieve the information about the energy-level structure of an absorbing medium by statistically analyzing twophoton transition probabilities obtained for different mutual time delays and signal-frequency chirps, using just a single nonlinear crystal.

\section{Acknowledgement}

This work was supported by the projects No. 18-22102S (J.P.) and 17-23005Y (J.S.) of the Czech Science Foundation. J.S. also acknowledges the Faculty of Science of Universidad de los Andes. R.J.L.M. thankfully acknowledges financial support by CONACYT under the project CB-2016-01/284372, and by DGAPAUNAM under the project UNAM-PAPIIT IA100718.

\section{References}

[1] B. E. A. Saleh, B. M. Jost, H.-B. Fei, M. C. Teich, Entangled-photon virtual-state spectroscopy, Phys. Rev. Lett. 80 (16) (1998) 3483.

[2] R. J. León-Montiel, J. Svozilik, L. J. Salazar-Serrano, J. P. Torres, Role of the spectral shape of quantum correlations in two-photon virtual-state spectroscopy, New. J. Phys. 15 (5) (2013) 053023.

[3] F. Schlawin, Entangled photon spectroscopy, J. Phys. B 50 (2017) 203001.

[4] A. K. Ekert, Quantum cryptography based on Bell's theorem, Phys. Rev. Lett. 67 (6) (1991) 661.

[5] M. A. Nielsen, I. L. Chuang, Quantum computation and quantum information, Cambridge Univ. Press, Cambridge, 2010.

[6] V. Giovannetti, S. Lloyd, L. Maccone, Quantum-enhanced measurements: beating the standard quantum limit, Science 306 (5700) (2004) 1330.

[7] D. I. Lee, T. Goodson III, Entangled photon absorption in an organic porphyrin dendrimer, J. Phys. Chem. B 110 (51) (2006) 25582.

[8] A. R. Guzman, M. R. Harpham, O. Süzer, M. M. Haley, T. Goodson III., Spatial control of entangled two-photon absorption with organic chromophores, J. Am. Chem. Soc. 132 (23) (2010) 7840.

[9] O. Roslyak, S. Mukamel, Multidimensional pump-probe spectroscopy with entangled twin-photon states, Phys. Rev. A 79 (2009) 063409. doi:10.1103/PhysRevA.79.063409

[10] F. Schlawin, K. E. Dorfman, B. P. Fingerhut, S. Mukamel, Manipulation of two-photon-induced fluorescence spectra of chromophore aggregates with entangled photons: A simulation study, Phys. Rev. A 86 (2012) 023851. doi:10.1103/PhysRevA.86.023851 
[11] M. G. Raymer, A. H. Marcus, J. R. Widom, D. L. P. Vitullo, Entangled photon-pair two-dimensional fluorescence spectroscopy (EPP-2DFS), J. Phys. Chem. B 117 (2013) 15559.

[12] K. E. Dorfman, F. Schlawin, S. Mukamel, Nonlinear optical signals and spectroscopy with quantum light, Rev. Mod. Phys 88 (2016) 045008.

[13] J. P. Villabona-Monsalve, O. Calderón-Losada, M. Nuñez Portela, A. Valencia, Entangled two photon absorption cross section on the $808 \mathrm{~nm}$ region for the common dyes zinc tetraphenylporphyrin and rhodamine b, J. Phys. Chem. A 121 (41) (2017) 7869-7875.

[14] J. Javanainen, P. L. Gould, Linear intensity dependence of a two-photon transition rate, Phys. Rev. A 41 (1990) 5088.

[15] H.-B. Fei, B. M. Jost, S. Popescu, B. E. A. Saleh, M. C. Teich, Entanglement-induced two-photon transparency, Phys. Rev. Lett. 78 (9) (1997) 1679.

[16] F. Schlawin, K. E. Dorfman, B. P. Fingerhut, S. Mukamel, Suppression of population transport and control of exciton distributions by entangled photons, Nat. Commun. 4 (2013) 1782.

[17] M. Shapiro, P. Brumer, Generation and control of chains of entangled atomion pairs with quantum light, Phys. Rev. Lett. 106 (2011) 150501.

[18] M. Shapiro, P. Brumer, Quantum control of molecular processes, WileyVCH, Weinheim, 2012.

[19] J. Perina, Quantum statistics of linear and nonlinear optical phenomena, Kluwer, Boston, 1991.

[20] B. Dayan, Theory of two-photon interactions with broadband downconverted light and entangled photons, Phys. Rev. A 76 (4) (2007) 043813.

[21] H. Oka, Efficient selective two-photon excitation by tailored quantumcorrelated photons, Phys. Rev. A 81 (6) (2010) 063819.

[22] J. Peřina, B. E. A. Saleh, M. C. Teich, Multiphoton absorption cross section and virtual-state spectroscopy for the entangled n-photon state, Phys. Rev. A 57 (5) (1998) 3972.

[23] F. Schlawin, K. Dorfman, S. Mukamel, Pump-probe spectroscopy using quantum light with two-photon coincidence detection, Physical Review A 93 (2) (2016) 023807.

[24] B. W. Shore, Definition of virtual levels, Am. J. Phys. 47 (1979) 262.

[25] J. J. Sakurai, Modern Quantum Mechanics, MA: Addison-Wesley, Boston, 1994. 
[26] D.-I. Lee, T. Goodson, Entangled and correlated two-photon absorption effects of an organic material, Digest of the IEEE/LEOS Summer Topical Meetings 15-16 (2007) 219-220.

[27] J. Svozilík, J. Peřina Jr., Properties of entangled photon pairs generated in periodically poled nonlinear crystals, Phys. Rev. A 80 (2) (2009) 023819.

[28] J. Svozilík, M. Hendrych, A. S. Helmy, J. P. Torres, Generation of paired photons in a quantum separable state in bragg reflection waveguides, Opt. Express 19 (4) (2011) 3115.

[29] A. Vallés, M. Hendrych, J. Svozilík, R. Machulka, P. Abolghasem, D. Kang, B. J. Bijlani, A. S. Helmy, J. P. Torres, Generation of polarization-entangled photon pairs in a bragg reflection waveguide, Opt. Express 21 (9) (2013) 10841 .

[30] R. Machulka, J. Svozilík, J. Soubusta, J. Peřina Jr., O. Haderka, Spatial and spectral properties of fields generated by pulsed second-harmonic generation in a periodically poled potassium-titanyl-phosphate waveguide, Phys. Rev. A 87.

[31] A. Allevi, O. Jedrkiewicz, E. Brambilla, A. Gatti, J. Peřina Jr, O. Haderka, M. Bondani, Coherence properties of high-gain twin beams, Phys Rev. A 90 (6) (2014) 063812.

[32] A. M. Pérez, T. S. Iskhakov, P. Sharapova, S. Lemieux, O. V. Tikhonova, M. V. Chekhova, G. Leuchs, Bright squeezed-vacuum source with 1.1 spatial mode, Opt. Lett. 39 (2014) 2403.

[33] F. Schlawin, S. Mukamel, Photon statistics of intense entangled photon pulses, J. Phys. B 46 (17) (2013) 175502.

[34] O. Haderka, R. Machulka, J. Peřina, A. Allevi, M. Bondani, Spatial and spectral coherence in propagating high-intensity twin beams, Sci. Rep. 5 (2015) 14365.

[35] J. Peřina Jr, Coherence and dimensionality of intense spatiospectral twin beams, Phys. Rev. A 92 (1) (2015) 013833.

[36] J. Peřina Jr., O. Haderka, A. Allevi, M. Bondani, Internal dynamics of intense twin beams and their coherence, Sci. Rep. 6 (2016) 22320.

[37] J. Peřina Jr., Spatial, spectral and temporal coherence of ultra-intense twin beams, Phys. Rev. A 93 (2016) 013852.

[38] J. Svozilík, J. Peřina, R. de J. León-Montiel, Virtual-state spectroscopy with frequency-tailored intense entangled beams, J. Opt. Soc. Am. B 35 (2) (2018) 460-467. 
[39] S. Carrasco, J. P. Torres, L. Torner, A. Sergienko, B. E. A. Saleh, M. C. Teich, Spatial-to-spectral mapping in spontaneous parametric downconversion, Phys. Rev. A 70 (4) (2004) 043817.

[40] A. Valencia, A. Ceré, X. Shi, G. Molina-Terriza, J. P. Torres, Shaping the waveform of entangled photons, Phys. Rev. Lett. 99 (24) (2007) 243601.

[41] D. Meshulach, Y. Silberberg, Coherent quantum control of two-photon transitions by a femtosecond laser pulse, Nature 396 (6708) (1998) 239242.

[42] B. Dayan, A. Pe'er, A. A. Friesem, Y. Silberberg, Two photon absorption and coherent control with broadband down-converted light, Phys. Rev. Lett. 93 (2) (2004) 023005.

[43] A. Joseph, R. Andrews, Entanglement-enhanced two-photon absorption in spectral phase-modulated type-I parametric down-conversion, J. Opt. Soc. Am. B 32 (7) (2015) 1450-1455.

[44] J. Kojima, Q.-V. Nguyen, Entangled biphoton virtual-state spectroscopy of the $A^{2} \Sigma^{+}-X^{2} \Pi$ system of OH, Chem. Phys. Lett. 396 (4) (2004) 323.

[45] Y. Shen, Principles of Nonlinear Optics, Wiley-Interscience, New York, 1984.

[46] S. Mukamel, Principles of Nonlinear Optical Spectroscopy, Oxford series in optical and imaging sciences, Oxford University Press, Oxford, 1999.

[47] R. J. Glauber, in Quantum Optics and Electronics, edited by C. DeWitt, and C. Cohen-Tannoudji, Gordon and Breach Science Publishers, New York, 1965.

[48] L. Mandel, E. Wolf, Coherence properties of optical fields, Rev. Mod. Phys. 37 (1965) 231.

[49] W. Wasilewski, A. I. Lvovsky, K. Banaszek, C. Radzewicz, Pulsed squeezed light: Simultaneous squeezing of multiple modes, Phys. Rev. A 73 (6) (2006) 063819.

[50] B. Huttner, S. Serulnik, Y. Ben-Aryeh, Quantum analysis of light propagation in a parametric amplifier, Phys. Rev. A 42 (1990) 5594-5600.

[51] C. K. Law, I. A. Walmsley, J. H. Eberly, Continuous frequency entanglement: Effective finite hilbert space and entropy control, Phys. Rev. Lett. 84 (23) (2000) 5304.

[52] C. K. Law, J. H. Eberly, Analysis and interpretation of high transverse entanglement in optical parametric down conversion, Phys. Rev. Lett. 92 (12) (2004) 127903. 
[53] O. Roslyak, C. A. Marx, S. Mukamel, Nonlinear spectroscopy with entangled photons: Manipulating quantum pathways of matter, Phys. Rev. A 79 (3) (2009) 033832. 